\title{
Proposta de uma metodologia para avaliação de defeitos de soldadura na manutenção de equipamentos sob pressão
}

\author{
(proposed methodology to evaluate welding defects during maintenance of equipments under pressure)
}

\author{
Paulo Silva $^{1}$, R. M. Miranda ${ }^{2, a}$, L. Quintino ${ }^{3,4}$ \\ ${ }^{1} I S Q$ - Instituto de Soldadura e Qualidade, Av. Prof. Dr. Cavaco Silva, nº33 - Taguspark-Oeiras 2740-120 Porto Salvo, Portugal. \\ ${ }^{2}$ UNIDEMI, Departamento de Engenharia Mecânica e Industrial, Faculdade de Ciências e Tecnologia, FCT, Universidade Nova de \\ Lisboa, 2829-516 Caparica, Portugal \\ rmiranda@fct.unl.pt \\ ${ }^{3}$ IST-UTL Instituto Superior Técnico, Lisboa, Portugal \\ ${ }^{4}$ IDMEC, Institute of Mechanical Engineering, TULISBON, Lisboa, Portugal
}

\begin{abstract}
Resumo
A utilização de técnicas avançadas de controlo não destrutivo como o Time of Flight Detection (ToFD) e o Phased Array para inspeção em manutenção de reservatórios de pressão permite a caracterização de numerosos defeitos não detectados durante a construção, como defeitos planares, cuja detecção por radiografia depende da sua orientação. Estas técnicas têm conduzido a um aumento substancial da fiabilidade dos equipamentos, uma vez que a detecção de defeitos num estágio inicial de desenvolvimento permite planear as intervenções, reduzir paragens e tempos não produtivos. Os defeitos de fabrico são identificados nas inspeções de manutenção pelo fato das técnicas usadas serem mais precisas e as áreas inspecionadas maiores do que as requeridas após construção. Alguns defeitos são facilmente classificados como tendo origem no fabrico e construção, mas outros não conseguem distinguir-se dos provocados pelas condições de serviço. Neste sentido, a decisão importante a tomar é a de avaliar se a evolução do defeito é possível de ocorrer, baseada no conhecimento das condições de serviço, do material, da dimensão e localização do defeito. Este artigo pretende fazer uma proposta de metodologia de avaliação de defeitos baseada num conjunto substancial de casos industriais de modo a apoiar o pessoal técnico nas fases de decisão em manutenção, inspeção e reparação de defeitos em juntas soldadas em reservatórios de pressão de processo.
\end{abstract}

Palavras chave: reservatórios de pressão, CND, manutenção, reparação, metodologias de aptidão para o uso, inspecção baseada no risco

Abstract: The use of advanced non-destructive techniques like ToFD and Phased Array to perform maintenance inspections of pressure vessels enables the characterization of numerous defects not detectable during the construction, as planar defects, whose detection by radiography is highly dependent on its orientation. These techniques lead to a substantial increase in reliability of equipments, since, the early detection of defects under development allows the planning of interventions, reducing down times, or non-productive times, and the number of unscheduled shutdowns. The deficiencies of fabrication are usually identified in maintenance inspections, because the techniques used are more sensitive and the tested areas are more extensive than required after construction. Some defects can be easily classified as manufacturing defects, but some are indistinguishable from the ones related to service conditions. Regarding its origin, the critical decision to take is to assess if future evolution is likely to occur, based on the application, material, service conditions and size of defect. This study envisages a proposal of a methodology for technical evaluation based on a large amount of industrial cases, aiming to assist personnel in decision steps in maintenance, inspection and repair of weld defects detected on pressure vessels from process.

Key words: pressure vessels, NDT, maintenance, repair, fitness for service methodologies, risk based inspection

\section{Equipamentos sob pressão}

A definição de Equipamento Sob Pressão não é uma matéria consensual. Devido à forma como diversas directivas, leis, regulamentos, normas, códigos, etc., são estruturadas é comum a existência de diferenças e contradições em relação ao significado de alguns termos técnicos.

(Recebido em 31/01/2011; Texto final em 10/06/2011).
Actualmente, a construção de ESP não afectos ao transporte, encontra-se regulamentada na comunidade Europeia através da transposição para a legislação interna de cada estado membro das seguintes directivas:

$\rightarrow$ "Directive 87/404/EEC - Simple Pressure Vessels Directive", $\rightarrow$ “Directive 97/23/EC - Pressure Equipment Directive».

A directiva 87/404/EEC aplica-se á produção em série de ESP não sujeitos a chama através de construção soldada com o objectivo de armazenamento de ar ou azoto a uma pressão 
superior a 0,5 bar. Define as exigências essenciais de segurança que os recipientes sob pressão simples devem satisfazer os procedimentos adequados à certificação e ao controlo da conformidade dos mesmos. Esta directiva foi transposta para direito nacional através do Dec. Lei n. ${ }^{\circ}$ 103/92 de 30 de Maio.

A directiva 97/23/EC estabelece as regras a que devem obedecer o projecto, o fabrico e a avaliação da conformidade, a comercialização e a colocação em serviço dos EPS/s. Aplica-se aos equipamentos sob pressão e aos conjuntos sujeitos a uma pressão máxima admissível (PS) superior a 0,5 bar. A transposição para o ordenamento jurídico Português é efectuada pelo Dec. Lei n. ${ }^{\circ} 211 / 99$ de 14 de Junho. O regulamento de instalação, funcionamento, reparação e alteração de equipamentos sob pressão estabelecido pelo Dec. Lei 97/2000 aplica-se a todos os equipamentos sob pressão, estando excluídos da aplicação deste os ESP sobre os quais não se verifiquem todas as condições descritas no Tabela 1.

Para além de outras condições de segurança definidas no DL 97/2000, este estabelece a obrigatoriedade de realização de inspecções periódicas para verificação da condição dos ESP/s e condiciona a renovação da autorização de funcionamento de cada ESP aos resultados aceitáveis das mesmas. As exigências do mercado actual condicionam de sobremaneira a disponibilidade dos equipamentos para realização e cumprimento das actividades de manutenção e obrigações legais, promovendo desta forma a inclusão nos PIE/s de manutenção, técnicas de controlo não destrutivo:

$\rightarrow$ mais confiáveis,

$\rightarrow \quad$ com obtenção de resultados no menor espaço de tempo possível;

e que permitam que, outro tipo de trabalhos decorram em simultâneo sem incremento de perigo para a saúde dos intervenientes (caso de técnicas de inspecção como o ToFDscan e Phased Array em detrimento do controlo radiográfico).

\section{Normas e códigos de construção}

As várias normas e códigos de construção publicados descrevem regras para o projeto e construção de equipamentos sob pressão. Em contraste com algumas áreas da engenharia de outros tipos de equipamentos, estes fornecem uma cobertura praticamente global, desde:

- calculo/projecto (esforços de tensão; mecanismos de fadiga, fluência; materiais; etc.),

- fabricação (processos de soldadura e sua validação, regras de manuseamento e enformação de materiais, detalhes construtivos, etc),

- inspeção (técnicas de CND, procedimentos, extensão e critérios de aceitação, etc.);

- Até á descrição/atribuição de responsabilidades dos intervenientes (dono, construtor, inspector, etc.).

A maioria dos códigos internacionais de ESP desenvolveramse até um ponto em que existe um elevado grau de similaridade entre eles. Temas centrais como critérios de cálculo, métodos de fabricação, extensão de controlo e critérios de aceitação de defeitos encontram-se baseados em princípios idênticos (mas não totalmente iguais).

$\mathrm{Na}$ Europa actualmente é usual a adopção, (para além do cumprimentos de todos os requisitos essenciais de segurança descritos na PED), de códigos e normas de construção de equipamentos sob pressão, (não sujeitos a chama), tais como:

- ASME - Boiler And Pressure Vessel Code, Section VIII, Div.1 [1],

- ASME - Boiler And Pressure Vessel Code, Section VIII, Div.2 [2],

- PD 5500 - Specification for Unfired Fusion Welded Pressure Vessels [3],

- EN 13445 - Unfired Pressure Vessels [4],

- CODAP, Division 1 - Code for Construction of Unfired Pressure Vessels [5],

- CODAP, Division 2 - Code for Construction of Unfired Pressure Vessels [6],

- AD 2000 Code - Technical Rules for Pressure Vessels [7],

- TEMA Standards - Standards of the Tubular Exchanger Manufacturers association [8].

Pese embora actualmente as normas europeias harmonizadas tenham passado a ser fortemente implantadas com a transposição da PED, ainda subsistem vários ramos da indústria que se

Tabela 1 - Âmbito do Decreto Lei 97/2000

\begin{tabular}{|l|l|}
\hline \multicolumn{1}{|c|}{ TIPO DE EQUIPAMENTO } & \multicolumn{1}{|c|}{} \\
\cline { 1 - 2 } Geradores de vapor de água ou de água sobreaquecida & $\begin{array}{l}\text { PS }>0,5 \text { bar } \\
\text { PS.V }>200 \text { bar.L } \\
\text { T }>110^{\circ} \mathrm{C}\end{array}$ \\
\hline Outros equipamentos de vapor de água ou de água sobreaquecida & $\begin{array}{l}\text { PS }>2 \text { bar } \\
\text { PS.V }>1000 \text { bar.L } \\
\text { T }>130^{\circ} \mathrm{C}\end{array}$ \\
\hline Caldeiras de fluido térmico & $\begin{array}{l}\text { PS }>2 \text { bar } \\
\text { PS.V }>500 \text { bar.L } \\
\text { T }>125^{\circ} \mathrm{C}\end{array}$ \\
\hline Tubagens & $\begin{array}{l}\text { PS } \geq 4 \text { bar } \\
\text { PS. } \varnothing \text { int. } \geq 1000\end{array}$ \\
\hline Outros equipamentos & $\begin{array}{l}\text { PS } \geq 2 \text { bar } \\
\text { PS.V } \geq 3000 \text { bar.L }\end{array}$ \\
\hline
\end{tabular}


Tabela 2 - Principais vantagens e limitação da técnica ToFD

\begin{tabular}{|c|c|}
\hline Vantagens & 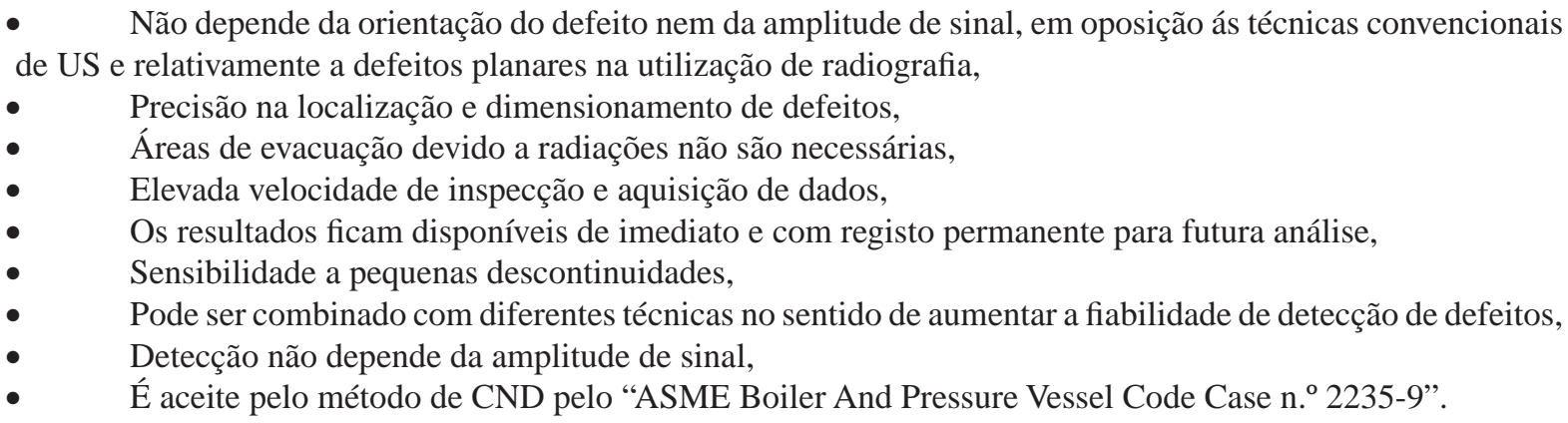 \\
\hline Limitações & $\begin{array}{l}\text { - Existência de uma zona morta de detecção em ambas as superfícies, } \\
\text { - Requer operadores experientes e especializados para interpretação, } \\
\text { - A geometria da área de análise e deficiências de acoplamento podem impedir a propagação da onda lateral, } \\
\text { - O sinal é sensível a material com grão grosseiro, }\end{array}$ \\
\hline
\end{tabular}

Tabela 3 - Principais vantagens e limitação da técnica Phased Array.

\begin{tabular}{|l|l|}
\hline Vantagens & • Redução dos tempos de inspecção, \\
& • Precisão na localização e dimensionamento de defeitos, \\
& • Áreas de evacuação devido a radiações não são necessárias, \\
& • Elevada velocidade de inspecção e aquisição de dados, \\
& - Sensibilidade a pequenas descontinuidades, \\
& - Sondagem utilizando múltiplos ângulos, \\
& • Possibilidade de registo permanente para futura análise, \\
\hline Limitações & - Requer operadores experientes e especializados para interpretação, \\
\hline
\end{tabular}

Tabela 4 - Metodologias de avaliação disponíveis no API 579 [14].

\begin{tabular}{|c|c|}
\hline \multicolumn{2}{|c|}{ Procedimentos para avaliação } \\
\hline \multirow{11}{*}{ Mecanismo de degradação e/ou defeitos pré-existentes } & Fractura frágil \\
\hline & Perda generalizada de espessura \\
\hline & Redução localizada de espessura \\
\hline & Corrosão por "pitting" \\
\hline & Danos por hidrogénio (“blisters", HIC e SOHIC) \\
\hline & Desalinhamento de soldadura e deformações \\
\hline & Defeitos tipo fissura (planares) \\
\hline & Operação em regime de fluência \\
\hline & Danos causados por incêndio \\
\hline & Cavidades e arrancamentos ou combinação de ambos \\
\hline & Defeitos de laminagem \\
\hline
\end{tabular}

mantêm fiéis á adopção de códigos, regras e recomendações ASME, como sejam as indústrias petrolíferas e petroquímicas [9].

Em conclusão verifica-se uma alteração pouco significativa entre as duas edições. mantendo-se como ensaio requerido o controlo radiográfico e adicionalmente o controlo por ultra-sons [10].

\section{Desenvolvimento de técnicas de CND por US}

Os desenvolvimentos associados ao controlo por US são muito vastos e incluem técnicas como: EMAT, "Guided Waves", ToFD, "Phase Array" [11-13]. De entre estas as últimas, duas são as mais promissoras em termos industriais no controlo de defeitos de soldadura em PEDs. Nas Tabelas 2 e 3 apresentamse esquematicamente as principais vantagens e limitações das técnicas ToFD e phase array, respectivamente. 


\section{Metodologias de apoio à decisão}

Nas últimas décadas foram desenvolvidas para a indústria diversas metodologias de apoio á decisão na avaliação de defeitos planares, todas elas tendo por base conceitos de mecânica da fractura. Assim, existem actualmente diversas abordagens aplicáveis a reservatórios, estruturas, nas indústrias de processo, aeronáuticas, nucleares, "off-shore", etc. de entre estas a "Fitness-For-Service", API 579-1/ASME FFS1, American Petroleum Institute and The American Society of Mechanical Engineers, Washington, D.C. [14] é talvez a mais aplicada industrialmente, em particular na avaliação de defeitos planares (nível 1). As metodologias "Fitness-For-Service" (FFS), são avaliações de engenharia quantitativas, realizadas para demonstrar a integridade estrutural em serviço de um componente contendo um defeito ou dano. Este documento fornece orientação na realização de avaliações de adequação ao uso (FFS) usando metodologias especificamente preparadas para equipamentos sob pressão. Estas orientações poderão ser usadas como apoio na tomada das decisões de reparação, substituição ou reposição em serviço com segurança e durante certo período de tempo de equipamentos sob pressão com defeitos detectados em inspecções de manutenção.

O API 579-1/ASME FFS-1 contém metodologias de avaliação de FFS relativas a diversos mecanismos de degradação e defeitos pré-existentes que vulgarmente são encontrados após a entrada em serviço dos componentes (Tabela 4).

Para cada procedimento de avaliação FFS existem três níveis. Cada nível de avaliação proporciona um equilíbrio entre o conservadorismo, a quantidade de informações necessárias e a complexidade da análise a ser realizada. As avaliações de nível 1 são as mais conservativas mas podem ser desenvolvidas com uma quantidade mínima de informação sobre o equipamento e historial de inspecção.

Os procedimentos de avaliação incluídos no nível dois são destinados a fornecer uma avaliação mais detalhada produzindo resultados mais precisos que o nível anterior. A informação de inspecção é semelhante á utilizada no nível anterior no entanto os procedimentos de cálculo são mais detalhados e produzem resultados menos conservadores.

A avaliação de nível 3 tem como objectivo produzir resultados mais precisos e menos conservativos do que os anteriores. Requer informação mais detalhada e precisa de inspecção e do componente e é recomendável que a análise seja efectuada através de técnicas numéricas, como FEA (análise por elementos finitos) ou técnicas experimentais quando apropriadas.

A abordagem de análise de falhas (FAD - "Failure Assesment Diagram), FAD procura estabelecer um critério de aceitabilidade para um componente contendo defeitos planares, onde o mecanismo de propagação da fenda é avaliado por dois critérios distintos combinados: propagação frágil (comportamento elástico) ou por colapso plástico (comportamento elastoplastico). A propagação frágil ocorre usualmente em equipamentos construídos com materiais de baixa tenacidade, mesmo, com descontinuidades relativamente pequenas. $\mathrm{O}$ colapso plástico ocorre normalmente já com descontinuidades grandes em materiais com elevada tenacidade.

\section{Proposta de metodologia de avaliação}

Os equipamentos sob pressão representam uma das categorias de componentes de maior importância na indústria de processo (petróleo, refinarias, petroquímicas, industria química, etc.). Em geral, fazem parte de uma cadeia continua, formada por diversos equipamentos, que trabalham submetidos a condições operacionais rigorosas de forma ininterrupta durante largos períodos de tempo sem paragens para inspecção e/ou manutenção.

A paragem não programada ou a extensão destas, resulta em grandes perdas económicas e de produção. A ocorrência de falhas, por sua vez, podem conduzir a acidentes de proporções elevadas, danos materiais, ambientais e, à perda de vidas humanas.

Em oposição ao anteriormente praticado a visão moderna reconhece na inspecção e na manutenção actividades estratégicas de aumento de disponibilidade e fiabilidade dos equipamentos e diminuição de custos de não produtividade.

Os profissionais de manutenção e inspecção envolvidos na operação de ESP utilizam todas as técnicas e recursos disponíveis para mantê-los em condições de segurança e adequação ao serviço previsto, estendendo a sua vida útil e minimizando a possibilidade de ocorrência de falhas.

São utilizadas diversas técnicas e métodos na inspecção e monitorização da integridade de equipamentos industriais. A escolha da técnica mais adequada deve ser realizada em função dos mecanismos de degradação presentes, dos tipos e dimensão de defeitos que se pretende detectar e dos materiais, (base e de soldadura), utilizados na fabricação dos equipamentos.

Os códigos de projecto e construção comummente utilizados como critério em actividades de manutenção não consideram o facto de que os equipamentos se degradam durante o serviço e não admitem a presença de algumas descontinuidades/danos devidas à degradação ou à fabricação original que podem ser encontrados em inspecções posteriores. O facto é que muitos dos defeitos considerados inaceitáveis pelos códigos de construção são, na verdade irrelevantes para a integridade do equipamento, sem necessidade de reparação, uma vez que o conhecimento existente sob os mecanismos de propagação de defeitos, quer em termos de comportamento de materiais (mecânica de fractura), quer em termos de técnicas de monitorização em tempo real permitem garantir a segurança das instalações sem necessidade de intervir antecipadamente.

Efectivamente, as reparações de ESP são operações que pretendem recolocar os equipamentos em serviço em condições de segurança, admitindo um determinado risco que caracteriza a diferença entre um equipamento novo e um reparado. A experiência, no entanto, já revelou que reparação de defeitos por processos de soldadura pode introduzir novos defeitos, algumas das vezes mais críticos que os originais.

As deficiências de construção normalmente são detectados em inspecções de manutenção, muito porque as técnicas usadas são mais sensíveis e as zonas controladas são mais extensas do que o requerido na sua construção. Alguns danos conseguem ser facilmente classificados como tendo origem na construção, no entanto outros tornam-se indistinguíveis relativamente 


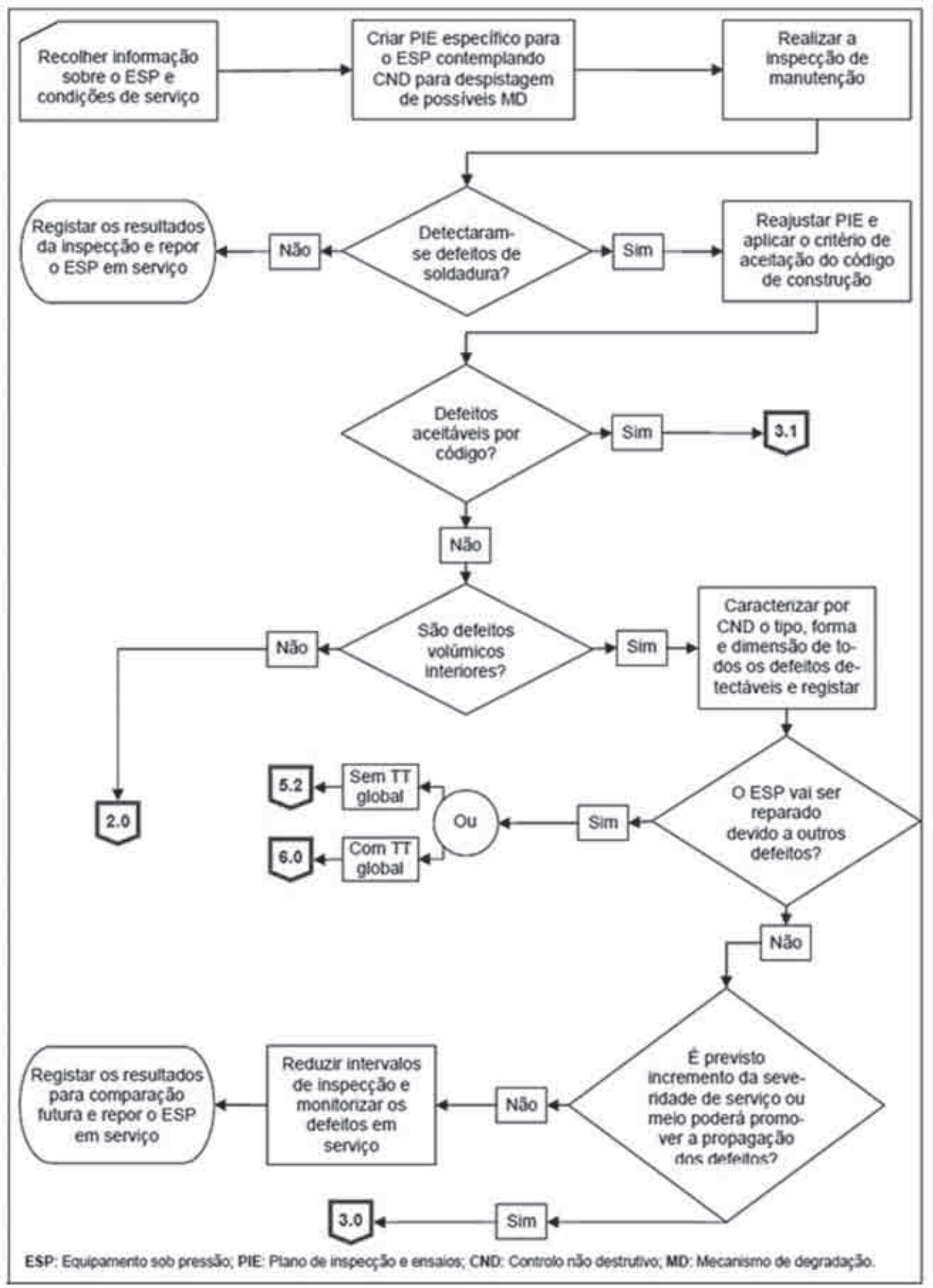

Figura 1 - Algoritmo de definição do plano de inspecção e ensaios, execução do controlo não destrutivo inicial e avaliação de defeitos volúmicos interiores. 


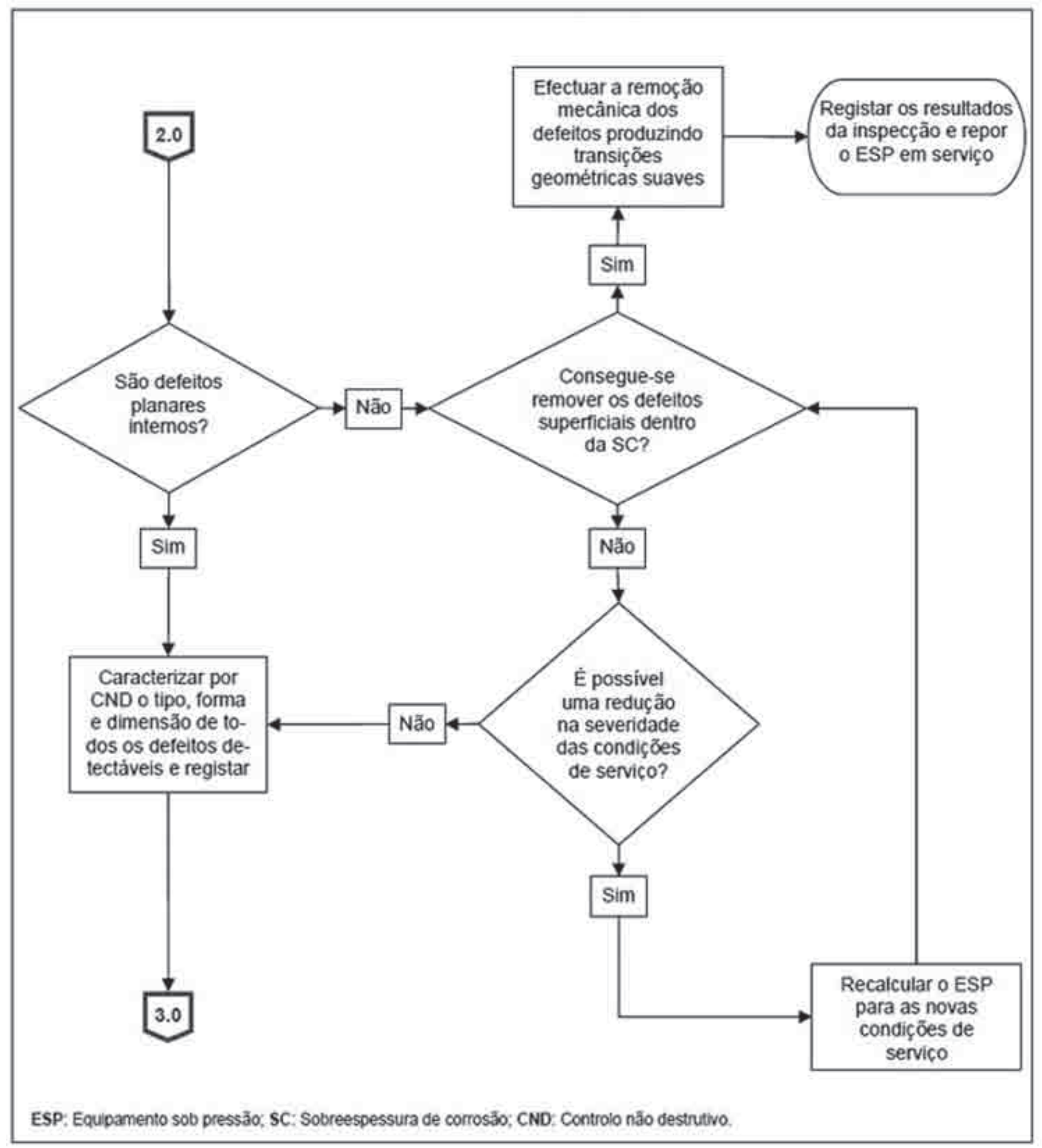

Figura 2 - Algoritmo de sequência de avaliação de defeitos superficiais 


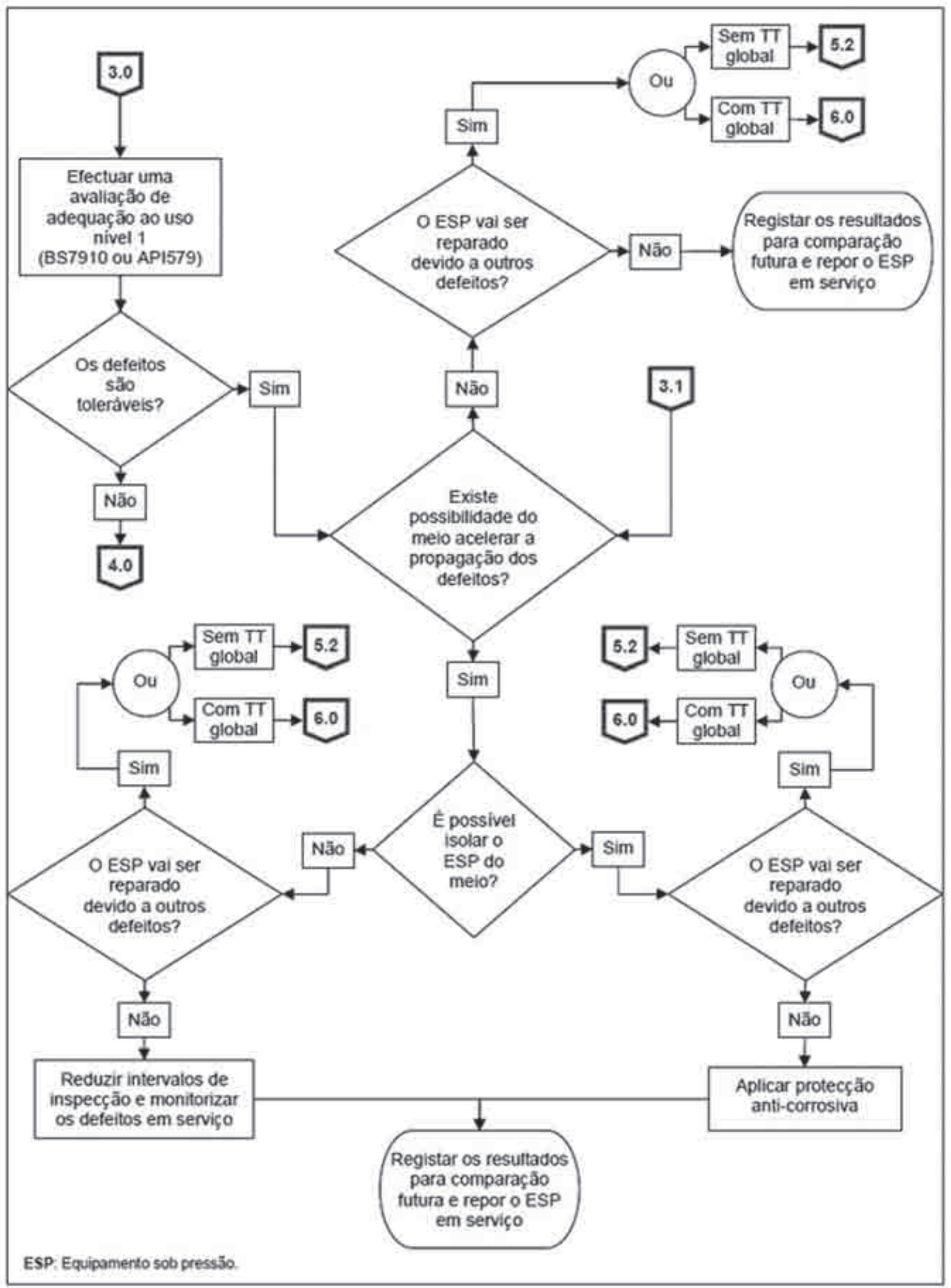

Figura 3 - Algoritmo de avaliação de defeitos planares internos 


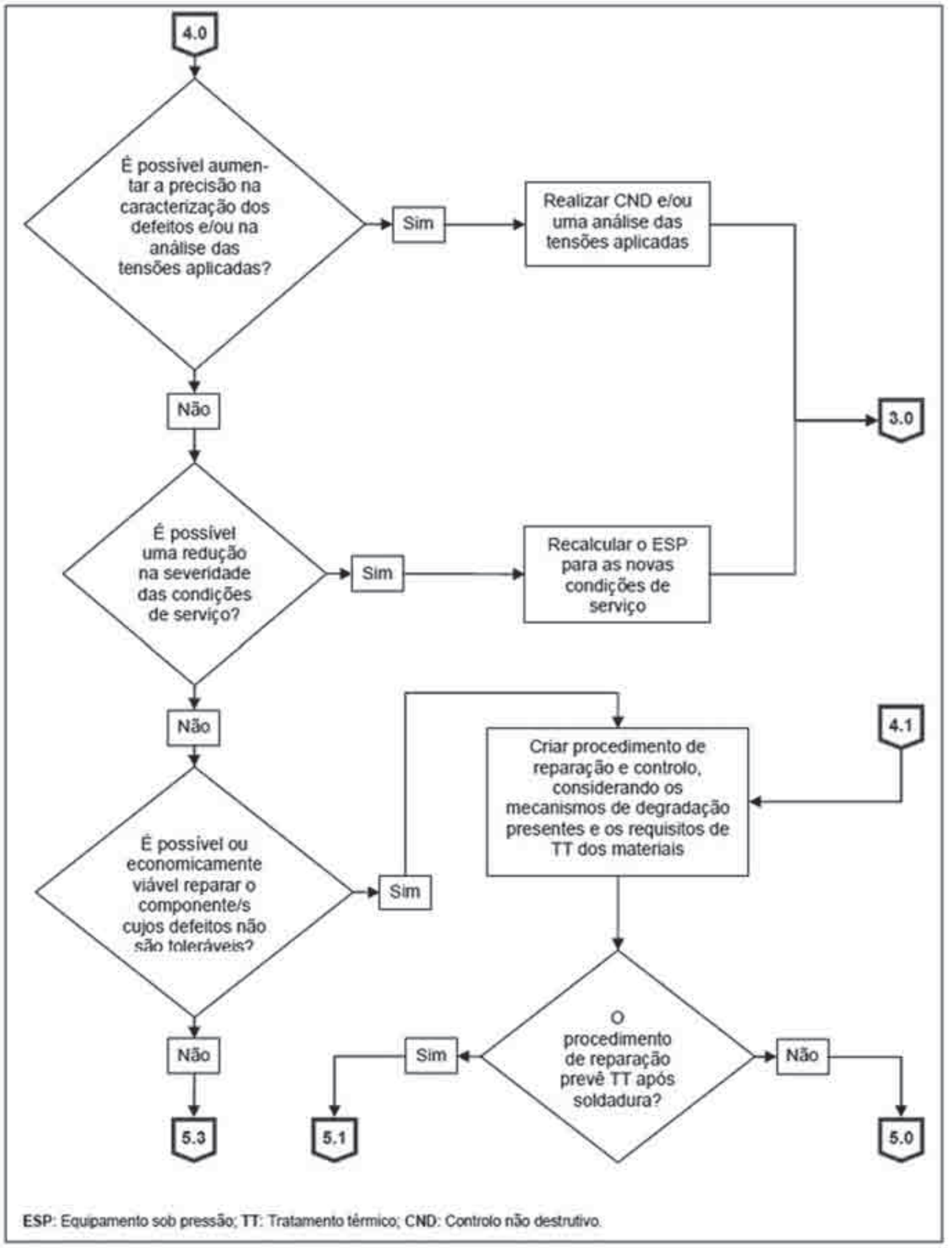

Figura 4 - Algoritmo de reavaliação detalhada dos defeitos planares internos através de métodos de caracterização dos defeitos e/ou uma redução na severidade das condições de serviço 


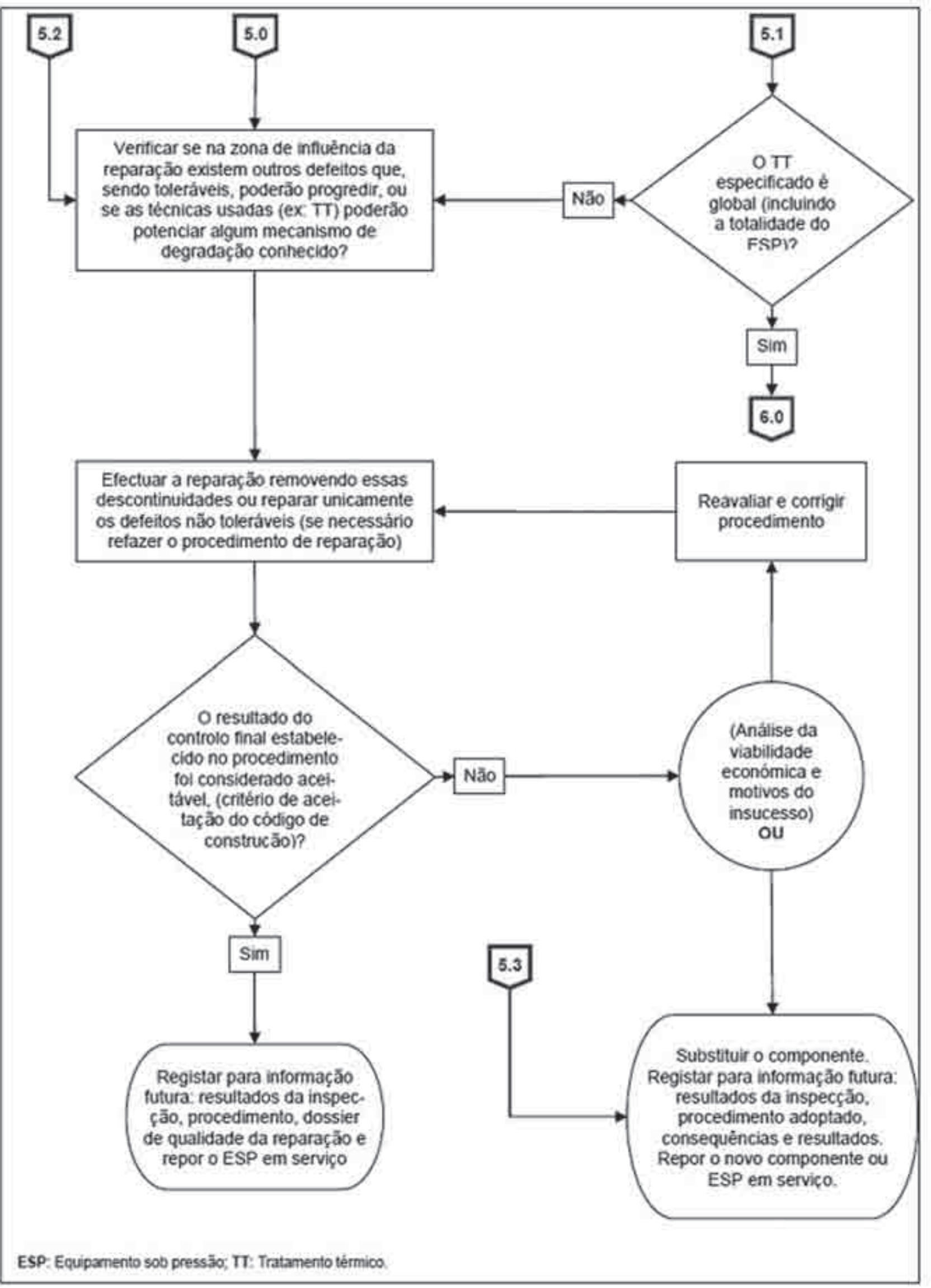

Figura 5 - Algoritmo de aferição da necessidade de tratamentos térmicos de relaxação de tensões localizados. 


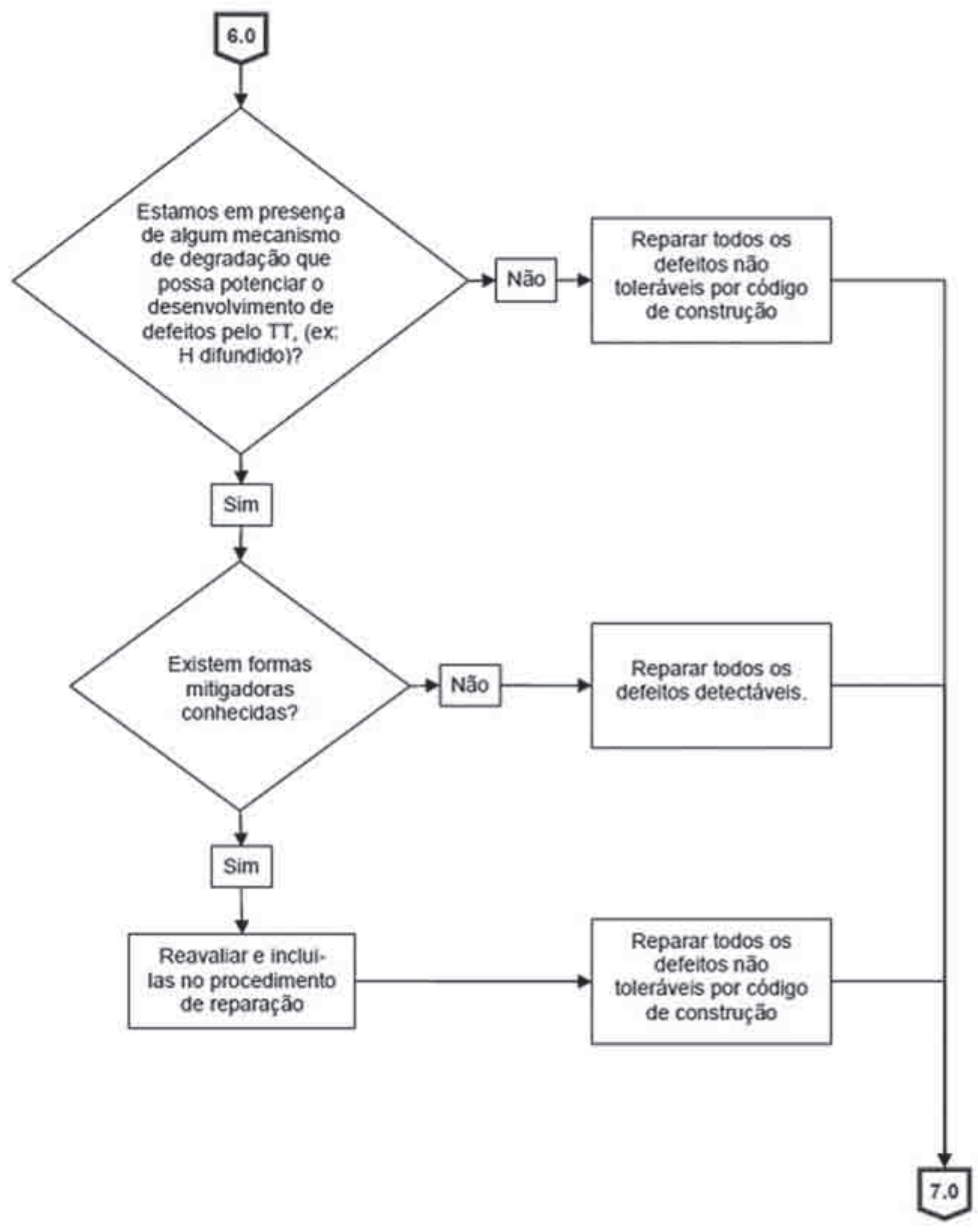

TT: Tratamento térmico, H: Hidrogónio.

Figura 6 - Algoritmo de aferição da necessidade de tratamentos térmicos de relaxação de tensões globais. 


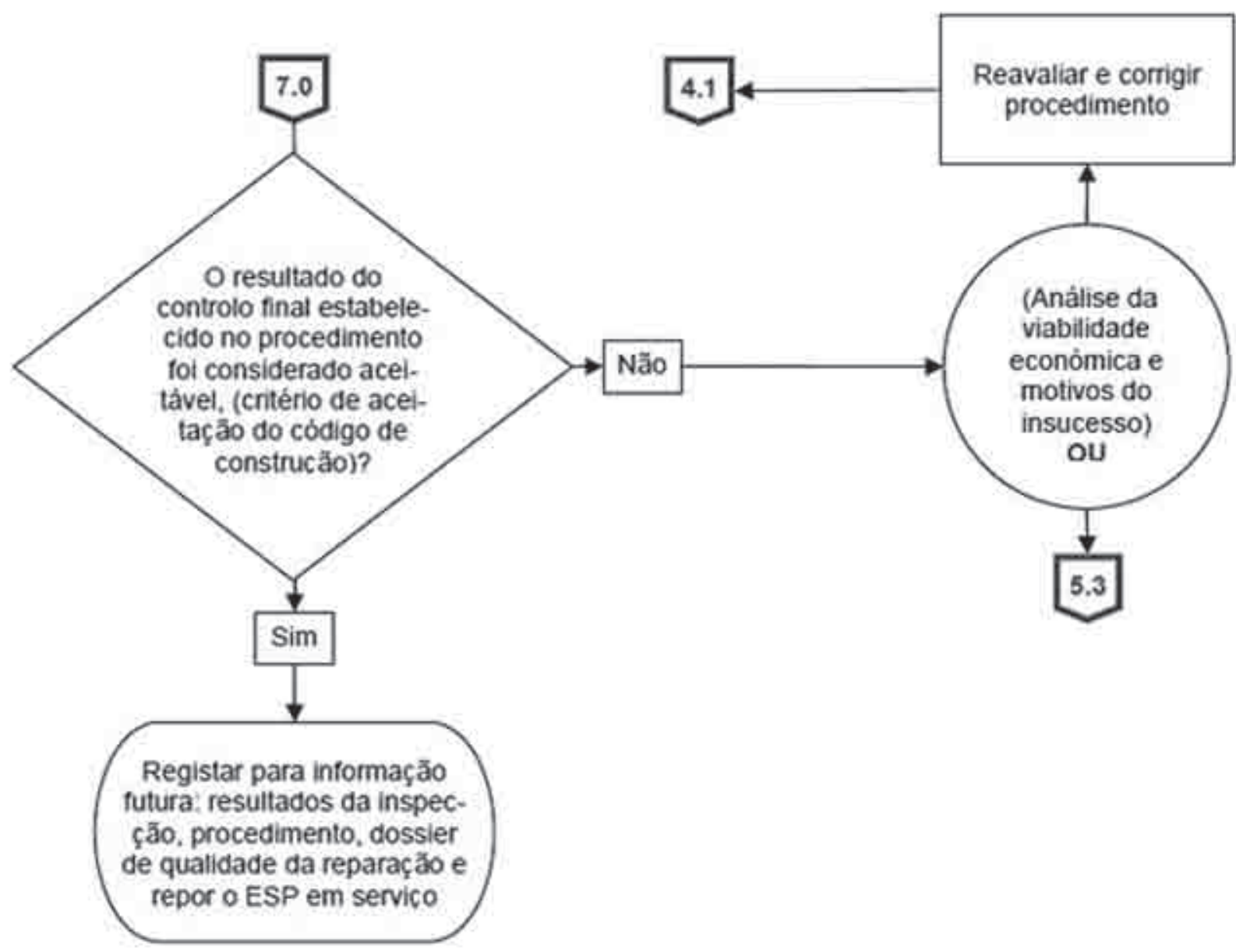

ESP: Equipamento sob pressão.

Figura 7 - Algoritmo de controlo final da reparação e aceitação do ESP para funcionamento

aos relacionados com as condições de serviço. Contudo a decisão fundamental que necessita sempre de ser tomada é, (independentemente da sua origem), se é provável a sua evolução futura baseada no material, solicitação, condições de serviço e dimensão do defeito.

As actividades de avaliação de integridade são multidisciplinares, envolvendo profissionais de inspecção de equipamentos, controlo não destrutivo, mecânica da fractura, análise de tensões, mecanismos de degradação, entre outros.

A metodologia proposta pretende agregar a multidisciplinaridade necessária à avaliação de defeitos de soldadura detectados em actividades de manutenção de equipamentos sob pressão. Sendo suportada em avaliações qualitativas de engenharia amplamente validadas, é intencionalmente abrangente e exaustiva, visando o apoio a profissionais da indústria na tomada de decisões em actividades de manutenção, inspecção e reparação de descontinuidades de soldadura.
Assim a metodologia foi estruturada em sete algoritmos (mostrados nas Figuras 1 a 7), sendo o primeiro, relativo à definição do plano de inspecção e ensaios, execução do controlo não destrutivo inicial e avaliação de defeitos volúmicos interiores.

No segundo algoritmo apresenta-se uma sequência de avaliação de defeitos superficiais e no terceiro uma primeira abordagem relativamente à avaliação de defeitos planares internos. Uma reavaliação mais detalhada dos defeitos planares internos, recorrendo, a uma maior precisão na sua caracterização dos defeitos e/ou uma redução na severidade das condições de serviço, é abordada no quarto algoritmo.

Os algoritmos quinto e sexto incidem respectivamente, nas precauções relativamente às reparações por soldadura com necessidade de tratamentos térmicos de relaxação de tensões localizados e globais. Por último, o sétimo algoritmo é dedicado ao controlo final da reparação e à aceitação do ESP para funcionamento. 


\section{Comentários finais}

Na sequência deste estudo verificou-se que os critérios de aceitação de soldadura dos códigos de projecto e construção são definidos num conjunto de pressupostos conservadores e genéricos. Relativamente a equipamentos sob pressão novos, isto é, em construção, apenas requerem uma comparação entre a dimensão das descontinuidades detectadas e a dimensão limite permitida independentemente da aplicação, solicitações e material do componente.

A manutenção de equipamentos sob pressão é uma actividade que envolve várias especialidades e a utilização conjunta do conhecimento de cada uma delas é imprescindível para o sucesso das actividades de avaliação e reparação por soldadura.

O reconhecimento de metodologias de avaliação de adequação ao uso como o "Fitness-For-Service" - API 5791/ASME FFS-1 ou o "Guide on Methods for Assesing the Acceptability of flaws in Fusion Welded Structures" - BS 7910, como ferramentas válidas e de carácter normativo, assumem papeis muito importantes na indústria contribuindo para o equilíbrio entre fiabilidade das unidades e custos de manutenção.

Os planos de inspecção e ensaios devem ser coerentes com o objectivo da inspecção, descriminando as técnicas de controlo não destrutivo adequadas à despistagem dos possíveis mecanismos de degradação presentes e capazes de detectar descontinuidades de tamanho inferior ao tamanho crítico. Os PIEs são instrumentos indispensáveis de controlo e acompanhamento da integridade dos equipamentos sob pressão, no entanto, deverão ser ferramentas dinâmicas e consecutivamente reavaliados e reajustados em função dos resultados obtidos.

Assim, elaborou-se uma metodologia de avaliação técnica de defeitos de soldadura em equipamentos sob pressão, suportada em considerações de carácter teórico multidisciplinar envolvendo conceitos de materiais, mecânica de fractura etc, e ainda nos códigos de construção aplicáveis nestes equipamentos.

Esta metodologia pretende ser um instrumento de apoio na tomada de decisões em actividades de manutenção, inspecção e reparação de defeitos detectados em equipamentos sob pressão na indústria de processo. Para isso tentou-se que esta contemplasse o mais exaustivamente possível, todas as situações expectáveis de ocorrer em termos de degradação e propagação de defeitos internos ou superficiais, volúmicos ou planares, decorrentes das acções de construção, serviço e manutenção em ESP.

\section{Referências Bibliográficas}

[1] "Rules for Construction of Pressure Vessels" ASME Boiler And Pressure Vessel Code, Section VIII, Div.1, ed. The American Society of Mechanical Engineers, 2007 Edition,

[2] "Rules for Construction of Pressure Vessels" ASME Boiler And Pressure Vessel Code, Section VIII, Div.2, ed. The American Society of Mechanical Engineers, 2007 Edition,

[3] "Specification for Unfired Fusion Welded Pressure Vessels" PD 5500:2006 -, ed. British Standards Institution, 2006 Edition, [4] "Unfired Pressure Vessels" - EN 13445 -, ed. Comité Européen de Normalisation, May 2002.
[5] "Code for Construction of Unfired Pressure Vessels" CODAP, Division 1 -, ed. SNCT publications, 2005 Edition.

[6] "Code for Construction of Unfired Pressure Vessels" CODAP, Division 2 -, ed. SNCT publications, 2005 Edition.

[7] "Technical Rules for Pressure Vessels" - AD 2000 Code ed, VdTUV, 2006.

[8] "Standards of the Tubular Exchanger Manufacturers association", Ninth Edition - ed. TEMA, 2007.

[9] "Rules for Construction of Pressure Vessels" - ANSI/ASME

- Boiler And Pressure Vessel Code, Section VIII, Div.1, ed. The American Society of Mechanical Engineers, 2007 Edition.

[10] "Use of Ultrasonic Examination in Lieu of Radiography Section I; Section VIII, Divisions 1 and 2; and Section XII" - Case 2235-9 - ed. The ASME Boiler and Pressure Vessel Standards Committee, 2005.

[11] Norbert Trimborn - "The Time-of-Flight-DiffractionTechnique" - NDTnet - September 1997, Vol.2 No.09

[12] Akihira NISHIKAWA, Noriyuki NAGAHAMA, Kouichi UMEDA, Tadamichi SAKAI, Shin-ichi OYA - "Industrial Applications of Ultrasonic Time-of-Flight-Diffraction (TOFD) Techniques for Various Field Targets" - ed. International Institute of Welding, Document No: V-1282-04, 2004.

[13] Liliana Silva, Pedro Barros, Rosa Miranda, Luísa Quintino - "Técnicas avançadas de inspecção não destrutiva" - ed. Tecnologia e Qualidade, 62/63, 2008, 35-41.

[14] “Fitness-For-Service", API 579-1/ASME FFS-1 - ed. American Petroleum Institute and The American Society of Mechanical Engineers, Washington, D.C., 2007, 